\title{
Skeletal muscle and whole body insulin resistance but not cardiac muscle insulin resistance could be improved by troglitazone therapy within 12 weeks in type-2 diabetes
}

\author{
Ikuo Yokoyama ${ }^{1}$, Toshiyuki Moritan ${ }^{2}$, Yusuke Inoue ${ }^{3}$ \\ ${ }^{1}$ Department of Cardiovascular Medicine, Clinical Research Center, Sanno Hospital of International University of Health and Welfare, \\ Tokyo, Japan \\ ${ }^{2}$ Department of Clinical Engineering, Faculty of Medical Engineering, Suzuka University of Medical Science, Suzuka, Japan \\ ${ }^{3}$ JapanDepartment of Radiology, Institute of Medical Science, Graduate School of Medicine University of Tokyo, Tokyo, Japan \\ Email: yokochan-tky@umin.ac.jp
}

Received 2 November 2012; revised 9 December 2012; accepted 17 November 2012

\begin{abstract}
Background: Existence of myocardial insulin resistance (IR) has been reported in type II diabetics (T2DM) and coronary artery disease (CAD). Improvement in heart and skeletal muscle IR after thiazolidinedione's therapy was reported in T2DM and CAD. However effects of troglitazone therapy (TRO) on myocardial IR remain uncertain. To clarify heart and skeletal muscle and whole body IR in T2DM without CAD by TRO to clarify whether TRO would provide different results. Methods: We analyzed data on 15 T2DM patients who underwent dynamic PET with ${ }^{18}$ F-FDG under insulin clamping before and during TRO (200 mg/day) and 17 controls. Results: Whole body glucose disposal rate (WBGR $\mathrm{mg} / \mathrm{min} / \mathrm{kg}$ ) in T2DM before TRO $(3.41 \pm 1.72)$ was significantly lower than in controls $(9.76 \pm 2.97, p<0.01)$ as was the skeletal muscle glucose utilization rate (SMGU $\mathrm{mg} / \mathrm{min} / \mathrm{kg})$; T2DM $(0.367 \pm 0.217)$ vs. controls $(1.34$ $\pm 0.613, \mathrm{p}<0.01)$ and myocardial glucose utilization rate $(\mathrm{MGU} \mathrm{mg} / \mathrm{min} / \mathrm{kg}$; T2DM $5.86 \pm 2.03 \mathrm{vs}$. controls $7.34 \pm 1.80, p<0.05$ ). WBGR in T2DM during TRO $(5.17 \pm 2.75, p<0.05)$ was significantly higher than that before TRO, as was the SMGU $(0.782 \pm 0.20, p<$ 0.05). The MGU in T2DM during TRO (6.59 \pm 0.72$)$ was comparable with that before TRO. Conclusion: Myocardial IR response to TRO differed from that in skeletal muscle and the whole body in T2DM without CAD.
\end{abstract}

Keywords: Insulin Resistance; Myocardial Insulin Resistance; Glucose Metabolism; Skeletal Muscle; Type II Diabetes Mellitus; ${ }^{18}$ F-FDG; PET

\section{INTRODUCTION}

Insulin resistance is defined as an impaired glucose utilization response to insulin stimulation and has been recognized to play an essential role in the development of a variety of metabolic diseases including type II diabetes mellitus (T2DM). Glucose is an essential substrate for the heart to maintain normal cardiac function of the failing heart due to ischemia; insulin resistance also plays a central role in the development of coronary artery disease (CAD) [1].

Positron emission tomography (PET) allows quantitative analysis of tissue metabolism and blood flow in vivo. PET has been used in research for the quantitative analysis of glucose utilization or insulin resistance in the heart and skeletal muscle in several diseases [2-5] using ${ }^{18}$ fluorine-fluoro-2-deoxy-D-glucose $\left({ }^{18} \mathrm{~F}\right.$-FDG). Thiazolidinediones have been used as oral hypoglycemic agents that act as insulin sensitizers. A positive effect of thiazolidinediones on improving both heart and skeletal muscle insulin resistance in patients with CAD and T2DM has been reported [6-8]. However, several antidiabetic medicines, including thiazolidinediones, could have different effects on the heart. For instance, it was reported that rosiglitazone was associated with a higher risk of a non-fatal acute myocardial infarction and heart failure than pioglitazone [9], implicating probable differences in the influence on the cardiovascular diseases between pioglitazone and rosiglitazone. Myocardial insulin resistance might have an important role in the myocardial metabolism of glucose, which in turn should play an important role in the ischemic but viable failing heart and the high mortality rate noted in persons with T2DM [10]. There are several reports on myocardial insulin resistance among patients with type-2 diabetes and coronary 
heart disorders. And based on any of the reports being made, it is clear that the coronary heart disorders significantly influence the myocardial insulin resistance. Most reports had used T2DM and CAD in the study of heart and skeletal muscle insulin resistance and their response to thiazolidinediones. However, myocardial glucose metabolism and/or myocardial insulin resistance varies according to the severity of myocardial ischemia, myocardial response to thiazolidinediones could not be constant in such variable status. Thus in this study, the target patients were selected for medical status without any coronary artery or heart disorders such to clearly filter and screen out the above possible influences and examine real myocardial glucose metabolism and myocardial insulin resistance. However, it is not clear if the effects of certain thiazolidinediones on insulin resistance differed among the heart and skeletal muscle and the whole body in patients with T2DM without a history of CAD. Indeed, insulin resistance in the heart during troglitazone therapy has not been reported. Therefore, this study aimed to test the hypothesis that the influences of troglitazone on insulin resistance differ among the heart and skeletal muscle and the whole body in patients with T2DM without a history of CAD. To test the hypothesis that TRO would provide different results than rosiglitazone and pioglitazone with respect to myocardial IR in T2DM without CAD, we aimed to clarify heart and skeletal muscle and whole body IR in T2DM without CAD over less than a 12-week treatment period by TRO.

\section{METHOD}

\subsection{Research Design and Methods}

\subsubsection{Patient Population}

We studied 15 asymptomatic patients with T2DM (mean age: $53.4 \pm 11.1 \mathrm{y}$; 10 males, 5 females) and 17 agematched healthy controls (mean age $53.6 \pm 9.48$ y; 14 males, 3 females). All study subjects were Japanese, and attended local health service centers or medical clinics. Nine of the 15 patients with T2DM had well-controlled essential hypertension. No patient was treated with medications for diabetes. Patients with ischemic heart disease were excluded by careful physical examination, echocardiography and treadmill testing by a specialist in cardiovascular medicine. Before enrollment, we informed all subjects of the nature of the study, after which they agreed to participate in the protocol, which was approved by the local Ethics Committee

\subsubsection{Study Design}

Troglitazone ( $200 \mathrm{mg} /$ day) was started 3 months after the initiation of priming therapy with conventional diet and mild-to-moderate exercise therapy (about 10000 walking steps/day) in all patients with T2DM. Physical examina- tion was performed every month during the study period. Exercise stress treadmill testing and resting echocardiography were performed before troglitazone therapy. Dynamic PET scan using ${ }^{18} \mathrm{~F}$-FDG under insulin clamping was performed before and during the therapy (14 patients were followed up $10-12$ weeks and only one patient was followed up 4 weeks.

\subsubsection{PET}

Preparation of ${ }^{18}$ fluorine-fluoro-2-deoxy-D-glucose $\left({ }^{18} \mathrm{~F}\right.$ $\mathrm{FDG})^{18} \mathrm{~F}$ was synthesized using the Cypris model 370 cyclotron (Sumitomo JYUKI Industries, Ltd., Kyoto, Japan), and ${ }^{18} \mathrm{~F}$-FDG was synthesized with an automated system based on the method reported by Ehrenkaufer et al. who developed that Fluorine-18 acetyl hypofluorite $\left({ }^{18} \mathrm{~F}\right.$-AcOF) was synthesized with a gas/solid-phase reaction. To react ${ }^{18} \mathrm{~F}$-AcOF directly with an unprotected sugar in $\mathrm{H}_{2} \mathrm{O}$ solution, ${ }^{18} \mathrm{~F}$-AcO in neon was bubbled into a dilute solution of D-glucal in $\mathrm{H}_{2} \mathrm{O}$ solution. $\mathrm{HCL}$ was added into the solution, which was warmed concisely. The solution was delivered through columns of charcoal, ion-retardation resin, and alumina to yield precursor of ${ }^{18}$ F-FDG [11]. Radiochemical purity was more than 95\%.

Acquisition of Myocardial Metabolic Images Myocardial ${ }^{18}$ F-FDG images were obtained using a Headtome IV PET scanner (Shimadzu Corp., Kyoto, Japan). This PET scanner has seven imaging planes; in-plane resolution is $4.5 \mathrm{~mm}$ at full width at half maximum (FWHM) and the z-axial resolution is $9.5 \mathrm{~mm}$ at FWHM. Effective inplane resolution was $7 \mathrm{~mm}$ after using a smoothing filter. The sensitivities of the Headtome IV scanners are 14 and $24 \mathrm{kcps}(\mu \mathrm{Ci} / \mathrm{ml})$ for direct and cross planes, respectively.

Following acquisition of transmission data for correct photon attenuation, we injected ${ }^{18} \mathrm{~F}$-FDG $(5$ - $10 \mathrm{mCi})$ and collected the dynamic PET data for 1 hour and 45 seconds. All medications and caffeine-containing foods were stopped 24 hours before the initiation of this study. During this interval, we obtained 19 dynamic scans using the following protocol: five 15 -second, three 30 -second, four 120 -second, four 300 -second and three 600 -second scans.

Quantification of Myocardial Glucose Utilization Rate (MGU) and Skeletal Muscle Glucose Utilization Rate (SMGU)

The amount of glucose metabolized by various organs was determined by calculating the tissue glucose utilization rate. Following the method previously reported by Ohtake et al. [12] we obtained input function from the time activity curve of the descending aorta corrected by 7 venous blood samplings. Using the input function, we determined $\mathrm{k}_{1} \times \mathrm{k}_{3} /\left(\mathrm{k}_{2}+\mathrm{k}_{3}\right)$ by Patlack graphic analysis and calculated both MGU and SMGU by substituting $\mathrm{k}_{1} \times$ $\mathrm{k}_{3} /\left(\mathrm{k}_{2}+\mathrm{k}_{3}\right)$ in the following equation.Tissue ${ }^{18} \mathrm{~F}$-FDG 
uptake rate $=\left\{\mathrm{k}_{1} \times \mathrm{k}_{3} /\left(\mathrm{k}_{2}+\mathrm{k}_{3}\right)\right\} \times\left\{\mathrm{BG}_{1}+\mathrm{BG}_{2}+\right.$ $\left.\mathrm{BG}_{3}\right\} / 3 / \mathrm{LC} \quad \mathrm{k}_{1}$ and $\mathrm{k}_{2}$ and $\mathrm{k}_{3}$ were rate constants in the following chemical formula.

$$
\begin{array}{ll}
\mathrm{k}_{1} & \mathrm{k}_{3} \text { Glu }(\mathrm{S}) \rightarrow \text { Glu }(\mathrm{T}) \rightarrow \text { Glu-6-phosphate }(\mathrm{T}) \\
\leftarrow \leftarrow & \\
\mathrm{k}_{2} & \mathrm{k}_{4} \text { Glu }=\text { glucose, } \mathrm{S}=\text { serum, } \mathrm{T}=\text { tissue, } \mathrm{k}_{4} \text { is }
\end{array}
$$
assumed to be zero in the myocardium and skeletal muscle. $\mathrm{BG}_{1}, \mathrm{BG}_{2}$ and $\mathrm{BG}_{3}$ were serum glucose concentrations during the dynamic PET scan using ${ }^{18}$ F-FDG. LC stands for Lumped Constant, which was calculated to be 1.0 in myocytes [13] and 1.2 in skeletal muscle cells, as repor- ted in human studies $[14,15]$.

All data were corrected for dead time effects to reduce error to less than $1 \%$. To avoid the influence of the partial volume effect associated with the object's size, recovery coefficients (RC) obtained from experimental phantom studies in our laboratory were used. The RC was 0.8 when myocardial wall thickness was $10 \mathrm{~mm}$. For the correction of the partial volume effect, wall thickness was measured with echocardiography by specialists in our hospital. The RC was taken into consideration in our program to measure the tissue glucose utilization rate.

We obtained MGU from the transaxial images. MGU was determined by averaging the above values. We also determined SMGU from the lumbar muscle [4]. To calculate the tissue glucose utilization rate, we used the SUN Ultra-30 high-speed image processing system (SUN Microsystems Japan Co., Ltd., Tokyo, Japan) with "Dr View" software (Asahi Kasei Information System Co., Ltd., Tokyo, Japan).

Data were collected following the use of the hyperinsulinemiceuglycemic clamp technique to achieve a steady state condition for determining the glucose consumption rate. A stable serum glucose concentration was maintained at $90-110 \mathrm{mg} / \mathrm{dl}$ by simultaneous infusion of regular insulin $(1 \mathrm{mIU} / \mathrm{min} / \mathrm{kg})$ at a fixed rate and a $20 \%$ glucose solution at a variable rate according to the glucose concentration. Whole body insulin resistance was measured by obtaining the glucose disposal rate during hyperinsulinemiceuglycemic clamping $(\mu \mathrm{mol} / \mathrm{min} / \mathrm{kg})$ according to a previously reported method [16].

\subsection{Statistical Analysis}

Values are expressed as the mean \pm standard deviation. Comparisons were made by one-way analysis of variance followed by Fisher's least significant difference tests for parametric distributions and the Kruskal-Wallis test followed by Scheffe's test for nonparametric distributions. A p value less than 0.05 was considered statistically significant.

\section{RESULT}

The whole body glucose disposal rate (WBGU) in pa- tients with T2DM was significantly lower than in control subjects. Before troglitazone therapy, SMGU and MGU in the T2DM patients were also significantly lower than in the control subjects. During troglitazone therapy, WBGU and SMGU were significantly higher than before the initiation of troglitazone therapy. However, MGU during hyperinsulinemiceuglycemic insulin clamping during troglitazone therapy was comparable to that before the therapy (Table 1).

Serum Glucose ConcentrationDuring hyperinsulinemiceuglycemic insulin clamping, the average serum glucose concentrations in patients with T2DM before therapy $(5.17 \pm 0.76 \mathrm{mmol} /$ liter $)$ and during therapy (5.21 \pm $0.87 \mathrm{mmol} /$ liter) were the same as in control subjects $(4.79 \pm 0.78 \mathrm{mmol} /$ liter$)$.

\subsection{Serum Insulin Concentration}

Serum insulin concentration in patients with T2DM under hyperinsulinemiceuglycemic clamping (63 \pm 24 $\mu \mathrm{U} / \mathrm{ml}$ ) was comparable to that in control subjects (61 \pm $29 \mu \mathrm{U} / \mathrm{ml})$. The serum insulin concentration at the beginning and at the end of the PET did not differ significantly. Plasma fasting insulin concentration in patients with T2DM $(8.0 \pm 3.1 \mu \mathrm{U} / \mathrm{ml})$ before therapy was significantly higher than in control subjects $(1.60 \pm 0.33$ $\mu \mathrm{U} / \mathrm{ml})$. This value during therapy $(7.5 \pm 4.1 \mu \mathrm{U} / \mathrm{ml})$ remained significantly higher than values for control subjects. There was no significant relationship between myocardial or SMGU and plasma insulin concentration in patients with T2DM.

\subsection{Plasma Free Fatty Acid Concentration (FFA)}

Plasma fasting FFA concentration in patients with T2DM was significantly higher than in control subjects; in

Table 1. Summary of main numerical data before and after troglitazone therapy.

\begin{tabular}{cccc}
\hline & \multicolumn{3}{c}{ Study subjects } \\
\cline { 2 - 4 } & $\begin{array}{c}\text { Type II } \\
\text { diabetes (pre) }\end{array}$ & $\begin{array}{c}\text { Type II } \\
\text { diabetes (post) }\end{array}$ & Controls \\
\hline WGDR & $3.41 \pm 1.72^{* *}$ & $5.17 \pm 2.75^{*}$ & $9.76 \pm 2.97$ \\
SMGU & $0.367 \pm 0.217^{* *}$ & $0.782 \pm 0.20^{*}$ & $1.34 \pm 0.613$ \\
MGU & $5.86 \pm 2.03^{\#}$ & $6.59 \pm 0.72$ & $7.34 \pm 1.80$ \\
Fasting FFA & $1.19 \pm 0.62^{* *}$ & $1.04 \pm 0.72^{* *}$ & $0.30 \pm 0.12$ \\
FFA-Insulin-CLAPING & $0.78 \pm 0.59^{\#}$ & $0.68 \pm 0.41^{\#}$ & $0.30 \pm 0.19$
\end{tabular}

p $<0.01$ vs. controls, ${ }^{*} \mathrm{p}<0.05$ vs. pre, ${ }^{*} \mathrm{p}<0.05$ vs. controlsWGDR, Whole body glucose disposal rate, SMGU; skeletal muscle glucose utilization rate during hyperinsulinemiceuglycemic insulin clamping, MGU; myocardial glucose utilization rate during hyperinsulinemiceuglycemic insulin clamping; FFA-Ins-CLAP, plasma free fatty acids during hyperinsulinemiceuglycemic insulin clamping (mEq/ liter). 
addition, this value during therapy was comparable with that before therapy. Serum FFA concentration during insulin clamping in patients with T2DM was significantly higher than in control subjects, but this value was not significantly reduced during therapy (Table 1).

\section{DISCUSSION}

This study evaluated MGU, SMGU and WBGU in patients with T2DM before and during troglitazone therapy. Prior to troglitazone treatment, WBGU, SMGU and MGU in patients with T2DM were significantly lower than in control subjects. Since all PET data and WBGU were collected under insulin clamping, these results showed that myocardial insulin resistance co-existed with skeletal muscle and whole body insulin resistance in patients with T2DM. Both WBGU and SMGU during the troglitazone therapy, that is, within 12 weeks of initiation of the treatment, were significantly higher than before troglitazone therapy. The SMGU was significantly increased by 2.13-fold and WBGU was 1.52-fold higher during than before the therapy. These data indicate that the primary role of troglitazone in the treatment of diabetes to improve whole body and skeletal muscle insulin resistance was accomplished within 12 weeks of therapy. Therefore, compliance with troglitazone therapy in this study was sufficient. However, MGU barely changed after the use of troglitazone in these T2DM patients (1.12-fold higher from baseline). These results show that troglitazone has little effect in improving myocardial insulin resistance in T2DM in contrast to the other thiazolidinediones (rosiglitazone [6,7] and pioglitazone [8]). It can be considered that the mechanisms for improving insulin resistance in the heart in response to thiazolidinediones differ among troglitazone, pioglitazone and rosiglitazone although all three agents had similar effects in improving skeletal muscle and whole body insulin resistance. This difference might also be due to the difference in the causes of heart, skeletal muscle and whole body insulin resistance. But the reason for the discrepant results cannot be explained in this study. In the current study, we used patients with T2DM without evidence of CAD to avoid the influence of myocardial ischemia to change myocardial glucose metabolism. Since subjects of the other studies [6-8] were those with T2DM and CAD, this difference in study subjects could be a factor for the discrepant results. A difference in the duration of thiazolidinedione therapy could also be a factor for the discrepancy in MGU response to thiazolidinediones; in our present study the subjects took the thiazolindedione 12 weeks or less while the period of dosage in the previous studies was 16 weeks. Another possible factor for the discrepancy is the racial differences between the two previous studies and our study. All of our subjects were
Japanese whereas those in the other two studies were from Western countries. Differences in gene regulation could be factor for the discrepancy since such a discrepancy has been reported between rosiglitazone and pioglitazone [17]. Interestingly, multicenter studies have shown that effects of thiazolidinediones on the prevention of cardiovascular events could differ between thiazolidiones (pioglitazone and rosiglitazone). For instance, favorable effects of pioglitazone in decreasing risk for non-fatal acute myocardial infarction and stroke were shown [9], a randomized placebo control trial revealed that pioglitazone reduced the complex of all-cause of death, non-fatal myocardial infarction, and stroke in T2DM patients who had CAD or peripheral obstructive vascular disease in persons whose HBA1c was more than $6.5 \%$ [18] and deleterious effects of rosiglitazone to increase risk of acute myocardial infarction [19], and higher risk of stroke, heart failure and death in elderly persons who used rosiglitazone in comparison with those using pioglitazone $[20,21]$ have been reported. Because of the difference in association with cardiovascular diseases or stroke between pioglitazone and rosiglitazone, we consider that myocardial responses to thiazolidiones could differ among troglitazone, pioglitazone and rosiglitazone. Accordingly, it can also be thought that since there is evidence that pioglitazone can prevent cardiovascular diseases and stroke and rosiglitazone can increase risk for cardiovascular diseases and stroke, troglitazone would have little apparent effect on the status of cardiovascular disease and myocardial metabolism. All of these are possible explanations for the discrepancy in this study.

Regarding the different response to troglitazone between heart and skeletal muscle, similarities and dissimilarities have been shown between myocardial glucose metabolism and skeletal muscle glucose metabolism. For instance myocardial insulin resistance was not seen in patients with essential hypertension although skeletal muscle and whole body insulin resistance existed [22]. Myocardial insulin resistance was also not detected in patients with T2DM and essential hypertension though skeletal muscle and whole body insulin resistance were present in T2DM and essential hypertension [3]. Such a dissimilarity might account for the discrepant response to troglitazone. However, much longer treatment periods would be required to improve myocardial insulin resistance with troglitazone.

The fact that WBGU and SMGU during troglitazone therapy significantly increased suggests that compliance with troglitazone therapy was sufficient in the present study. Therefore, the unchanged MGU under insulin clamping could be attributed to the unchanged plasma FFA levels under troglitazone therapy with good compliance. The reason why FFA levels were not changed 
significantly while WBGU and SMGU during troglitazone therapy were significantly increased can only be speculated upon. Possibly, the effects of troglitazone on skeletal muscle and whole body insulin resistance should not be attributed to FFA levels but to other factors such as adipocytes. Since troglitazone acts as a peroxisome proliferator-activated receptor- $\gamma$ (PPAR- $\gamma$ ) agonist [23, 24], activation of the PPAR- $\gamma$ in adipocytes leading to increase adipocytokine secretion, the reversal effect of troglitazone on the down regulatory effect on inflammatory cytokines [23] and the effect of increasing the number of small adipocytes by troglitazone [25] might be related to the improved skeletal muscle and whole body insulin resistance in patients with T2DM. However, effects of activation of PPAR- $\gamma$ and/or adipocytokines on myocardial insulin resistance remain uncertain. Basically insulin-sensitive myocardial glucose metabolism shifts from FFA metabolism after intake of carbohydrate-rich foods or insulin stimulation. Therefore, if insulin resistant in the heart was present, such a glucose-FFA cycle in the heart would be influenced in several diseases with insulin resistance. However, findings on insulin resistance in the heart are not consistent with studies of several diseases in which insulin resistance plays a central role. For example, in patients with T2DM, myocardial insulin resistance exists in patients without hypertension and off medications [5], but disappears in patients with T2DM and essential hypertension [3] and in patients who are treated with sulfonylurea drugs [26]. Myocardial insulin resistance was not decreased but increased in patients with essential hypertension [22] or hypertriglyceridemia without diabetes nor was it decreased in patients with T2DM and essential hypertension [27], although skeletal muscle and whole body insulin resistance clearly existed in such diseases. Such conflicting findings suggest that the myocardium has mechanisms to oppose insulin resistance in patients with whole body insulin resistance [3]. Nine T2DM patients with essential hypertension were included in this study; so such conflicting mechanisms with regard to the fact that myocardial insulin resistance disappeared in non-dibetic patients with essential hypertension could be related to our results that myocardial insulin resistance was unchanged during troglitazone therapy because myocardial insulin resistance disappeared in patients with T2DM and essential hypertension [3].

Because of the adverse events involving serious liver damage, troglitazone was withdrawn from the market. Our study was conducted before the withdrawal. The results of this study have limited direct relevance to clinical practice, However, these results should have value in investigating insulin resistance in T2DM and its response to thiazolidinedione treatment. Although we tried to select patients with a low probability of CAD based on the absence of signs or symptoms of myocardial ischemia, normal rest echocardiography, and normal symptom-limited treadmill test, these selection criteria could not completely exclude diabetic individuals with occult CAD. Since this study was an open trial, results should be verified by a double blind trial. However, due serious liver damage from troglitazone, a double blind trial is not feasible. These are the limitations of this study.

\section{CONCLUSION}

Troglitazone therapy within 12 weeks increased SMGU and the glucose disposal rate but not MGU under hyperinsulinemiceuglycemic insulin clamping in patients with T2DM. Therefore, the mechanisms causing or reversing myocardial insulin resistance could differ from those of skeletal muscle and whole body insulin resistance in patients with T2DM.

\section{COMPETING INTERESTS}

There are non-financial competing interests with this article.

\section{AUTHORS' CONTRIBUTIONS}

Dr. Yokoyamadesigned the study and recruited study subjects. Drs Yokoyama, Inoue, Moritanmade PET data sampling and data analysis. Drs. Inoue, Moritanprovided valuable discussion and advice in writing this manuscript.

\section{REFERENCES}

[1] Despres, J.P., Lamarche, B., Mauriège, P., Cantin, B., Dagenais, G.R., Moorjani, S. and Lupien, P.J. (1996) Hyperinsulinemia as an independent risk factor for ischemic heart disease. The New England Journal of Medicine, 334, 952-957. doi:10.1056/NEJM199604113341504

[2] Voipio-Pulkki, L.M., Nuutila, P., Knuuti, M.J., Ruotsalainen, U., Haaparanta, M., Teräs, M., Wegelius, U. and Koivisto, V.A.(1993) Heart and skeletal muscle glucose disposal in type 2 diabetic patients as determined by positron emission tomography. Journal of Nuclear Medicine, 34, 2064-2067.

[3] Yokoyama, I., Ohtake, T., Momomura, S., Yonekura, K., Yamada, N., Nishikawa, J., Sasaki, Y. and Omata, M. (1998) Organ-specific insulin resistance in patients with noninsulin-dependent diabetes mellitus and hypertension. Journal of Nuclear Medicine, 39, 884-889.

[4] Yokoyama, I., Yonekura, K., Moritan, T., Tateno, M., Momose, T., Ohtomo, K., Inoue, Y. and Nagai, R.(2001) Troglitazone improves whole-body insulin resistance and skeletal muscle glucose use in type II diabetic patients. Journal of Nuclear Medicine, 42, 1005-1010.

[5] Yokoyama, I., Yonekura, K., Ohtake, T., Kawamura, H., 
Matsumoto, A., Inoue, Y., Aoyagi, T., Sugiura, S., Omata, M., Ohtomo, K. and Nagai, R. (2000) Role of insulin resistance in heart and skeletal muscle F-18 fluorodeoxyglucose uptake in patients with non-insulin-dependent diabetes mellitus. Journal of Nuclear Cardiology, 7, 242248. doi:10.1016/S1071-3581(00)70013-4

[6] Hällsten, K., Virtanen, K.A., Lönnqvist, F., Janatuinen, T., Turiceanu, M., Rönnemaa, T., Viikari, J., Lehtimäki, T., Knuuti, J. and Nuutila, P. (2004) Enhancement of insulinstimulated myocardial glucose uptake in patients with Type 2 diabetes treated with rosiglitazone. Diabetic Medicine, 21, 1280-1287.

doi:10.1111/j.1464-5491.2004.01332.x

[7] Lautamaki, R., Airaksinen, K.E., Seppanen, M., Toikka, J., Luotolahti, M., Ball, E., Borra, R., Harkonen, R., Iozzo, P., Stewart, M., Knuuti, J. and Nuutila, P. (2005) Rosiglitazone improves myocardial glucose uptake in patients with type 2 diabetes and coronary artery disease: A 16week randomized, double-blind, placebo-controlled study. Diabetes, 54, 2787-2794. doi:10.2337/diabetes.54.9.2787

[8] Naoumova, R.P., Kindler, H., Leccisotti, L., Mongillo, M., Khan, M.T., Neuwirth, C., Seed, M., Holvoet, P., Betteridge, J. and Camici, P.G. (2007) Pioglitazone improves myocardial blood flow and glucose utilization in nondiabetic patients with combined hyperlipidemia: A randomized, double-blind, placebo-controlled study. Journal of the American College of Cardiology, 50, 20592060. doi:10.1016/j.jacc.2007.07.070

[9] Lincoff, A.M., Wolski, K., Nicholls, S.J. and Nissen, S.E. (2007) Pioglitazone and risk of cardiovascular events in patients with type 2 diabetes mellitus: A meta-analysis of randomized trials. Journal of the American Medical Association, 298, 1180-1188.

doi:10.1001/jama.298.10.1180

[10] Barsheshet, A., Shotan, A., Cohen, E., Garty, M., Goldenberg, I., Sandach, A., Behar, S., Zimlichman, E., Lewis, B.S., Gottlieb, S. and HFSIS Steering Committee and Investigators (2010) Predictors of long-term (4-year) mortality in elderly and young patients with acute heart failure. European Journal of Heart Failure, 12, 833-840. doi:10.1093/eurjhf/hfq079

[11] Ehrenkaufer, R.E., Potocki, J.F. and Jewett, D.M. (1989) Simple synthesis of F-18 labeled 2-fluoro-2-deoxy-D-glucose. Journal of Nuclear Medicine, 25, 333-337.

[12] Ohtake, T., Kosaka, N., Watanabe, T., Yokoyama, I., Moritan, T., Masuo, M., Iizuka, M., Kozeni, K., Momose, T., Oku, S., Sugimoto, T. and Iio, M. (1991) Noninvasive method to obtain input function for measuring tissue glucose utilization of thoracic and abdominal organs. Journal of Nuclear Medicine, 32, 1432-1438.

[13] Ng, C.K., Soufer, R. and McNulty, P.H. (1998) Effect of hyperinsulinemia on myocardial fluorine-18-FDG uptake. Journal of Nuclear Medicine, 39, 379-383.

[14] Kelly, D.E., Williams, K.V. and Goodpaster, B. (1999) Determination of the lumped constant for $\left[{ }^{18} \mathrm{~F}\right]$ fluorodeoxyglucose in human skeletal muscle. Journal of Nuclear Medicine, 40, 1798-1804.

[15] Peltoniemi, P., Lönnroth, P., Laine, H., Oikonen, V., Tol- vanen, T., Grönroos, T., Strindberg, L., Knuuti, J. and Nuutila, P. (2000) Lumped constant for $\left[{ }^{18} \mathrm{~F}\right]$ fluorodeoxyglucose in skeletal muscles of obese and nonobese humans. American Journal of Physiology-Endocrinology and Metabolism, 279, E1120-E1130.

[16] Yokoyama, I., Ohtake, T., Momomura, S., Yonekura, K., Shin, W.S., Nishikawa, J., Sasaki, Y. and Omata, M. (1998) Hyperglycemia rather than insulin resistance is related to coronary flow reserve in patients with non-insulin dependent diabetes mellitus. Diabetes, 47, 119-124. doi:10.2337/diabetes.47.1.119

[17] Hsiao, A., Worrall, D.S., Olefsky, J.M. and Subramaniam, S. (2004) Variance-modeled posterior inference of microarray data: Detecting gene-expression changes in 3T3-L1 adipocytes. Bioinformatics, 20, 3108-3127. doi:10.1093/bioinformatics/bth371

[18] Dormandy, J.A., Charbonnel, B., Eckland, D.J., Erdmann, E., Massi-Benedetti, M., Moules, I.K., Skene, A.M., Tan, M.H., Lefèbvre, P.J., Murray, G.D., Standl, E., Wilcox, R.G., Wilhelmsen, L., Betteridge, J., Birkeland, K., Golay, A., Heine, R.J., Korányi, L., Laakso, M., Mokán, M., Norkus, A., Pirags, V., Podar, T., Scheen, A., Scherbaum, W., Schernthaner, G., Schmitz, O., Skrha, J., Smith, U. and Taton, J. (2005) Secondary prevention of macrovascular events in patients with type 2 diabetes in the PROactive Study (PROspective pioglitAzone Clinical Trial in macroVascular Events): A randomised controlled trial. Lancet, 366, 1279-1289. doi:10.1016/S0140-6736(05)67528-9

[19] Nissen, S.E. and Wolski, K. (2007) Effect of rosiglitazone on the risk of myocardial infarction and death from cardiovascular causes. The New England Journal of Medicine, 356, 2457-2471. doi:10.1056/NEJMoa072761

[20] Juurlinkm, D.N., Gomes, T., Lipscombe, L.L., Austin, P.C., Hux, J.E. and Mamdani, M.M. (2009) Adverse cardiovascular events during treatment with pioglitazone and rosiglitazone: Population based cohort study. British Medical Journal, 339, b2942. doi:10.1136/bmj.b2942

[21] Graham, D.J., Ouellet-Hellstrom, R., MaCurdy, T.E., Ali, F., Sholley, C., Worrall, C. and Kelman, J.A. (2010) Risk of acute myocardial infarction, stroke, heart failure, and death in elderly medicare patients treated with rosiglitazone or pioglitazone. Journal of the American Medical Association, 3044, 411-418. doi:10.1001/jama.2010.920

[22] Nuutila, P., Maeki, M., Laine, H., Knuuti, J., Ruotsalainen, U., Kuotohahiti, M., Haaparanta, M., Solin, O., Jula, A., Koivisto, V.A., Voipio-Pulkki, L.M. and Yki-jaeynen, H. (1995) Insulin action on heart and skeletal muscle glucose uptake in essential hypertension. Journal of Clinical Investigation, 96, 1003-1009. doi:10.1172/JCI118085

[23] Tanaka, T., Itoh, H., Doi, K., Fukunaga, Y., Hosoda, K., Shintani, M., Yamashita, J., Chun, T.H., Inoue, M., Masatsugu, K., Sawada, N., Saito, T., Inoue, G., Nishimura, H., Yoshimasa, Y. and Nakao, K. (1999) Down regulation of peroxisome proliferator-activated receptor gamma expression by inflammatory cytokines and its reversal by thiazolidinediones. Diabetologia, 42, 702-710. doi:10.1007/s001250051218

[24] De Vos, P., Lefebvre, A.M., Miller, S.G., Guerre-Millo, 
M., Wong, K., Saladin, R., Hamann, L.G., Staels, B., Briggs, M.R. and Auwerx, J. (1996) Thiazolidinediones repress ob gene expression in rodents via activation of peroxisome proliferator-activated receptor gamma. Journal of Clinical Investigation, 98, 1004-1009.

doi:10.1172/JCI118860

[25] Okuno, A., Tamemoto, H., Tobe, K., Ueki, K., Mori, Y., Iwamoto, K., Umesono, K., Akanuma, Y., Fujiwara, T., Horikoshi, H., Yazaki, Y. and Kadowaki, T. (1998) Troglitazone increases the number of small adipocytes without the change of white adipose tissue mass in obese Zucker rats. Journal of Clinical Investigation, 101, 1354-1361. $\underline{\text { doi:10.1172/JCI1235 }}$
[26] Yokoyama, I., Inoue, Y., Moritan, T., Ohtomo, K. and Nagai, R. (2006) Myocardial glucose utilisation in type II diabetes mellitus patients treated with sulphonylurea drugs. European Journal of Nuclear Medicine and Molecular, 33, 703-708.

[27] Yokoyama, I., Ohtake, T., Momomura, S., Yonekura, K., Kobayakawa, N., Aoyagi, T., Sugiura, S., Yamada, N., Ohtomo, K., Sasaki, Y., Omata, M. and Yazaki, Y. (1999) Insulin action on Heart and skeletal muscle FDG uptake in patients with hypertriglyceridemia. Journal of Nuclear Medicine, 40, 1116-1121. 